Annals of Funct. Anal. 5 (2014), no. 1, 36-50
unctional ISSN: 2008-8752 (electronic)
nnalysis

\title{
KREIN SPACE NUMERICAL RANGES: COMPRESSIONS AND DILATIONS
}

\author{
N. BEBIANO ${ }^{1 *}$ AND J. DA PROVIDENCIA $^{2}$ \\ Dedicated to Professor T. Ando, in recognition of his outstanding mathematical achievements \\ Communicated by K. S. Berenhaut
}

\begin{abstract}
A criterion for the numerical range of a linear operator acting in a Krein space to be a two-component hyperbolical disc is given, using the concept of support function. A characterization of the Krein space numerical range as a union of hyperbolical discs is obtained by a reduction to the two-dimensional case. We revisit a famous result of Ando concerning the inclusion relation $W(A) \subseteq W(B)$ of the numerical ranges of two operators $A$ and $B$ acting in (possibly different) Hilbert spaces, and the condition that $A$ can be dilated to an operator of the form $B \otimes I$. The extension of this result to operators acting in Krein spaces is investigated.
\end{abstract}

\section{INTRODUCTION}

Let $\mathcal{H}$ be a Hilbert space. Suppose $J$ is an involutive self-adjoint operator acting on $(\mathcal{H},\langle.,\rangle$.$) . Then \mathcal{H}$ can be viewed as a (complex) Krein space with respect to the indefinite inner product $[x, y]:=\langle J x, y\rangle$ for any $x, y \in \mathcal{H}$, and $J$ is the so-called metric matrix. For a detailed study of Krein spaces and operators therein we refer the interested reader to $[1,10]$. In our discussion, we will identify $\mathcal{H}$ with $\mathbb{C}^{n}$ if $\mathcal{H}$ has dimension $n$. In such a case, we will identify $\mathcal{B}(\mathcal{H})$, the algebra of all bounded linear operators on $\mathcal{H}$, with the algebra of $n \times n$ complex matrices $M_{n}$. The Krein space numerical range of an operator $A \in \mathcal{B}(\mathcal{H})$ is

Date: Received: 17 May 2013; Accepted: 6 June 2013.

* Corresponding author.

2010 Mathematics Subject Classification. Primary 15A60; Secondary 46C20, 15A63.

Key words and phrases. Krein space, indefinite inner product space, numerical range, compression, dilation. 
defined as

$$
W_{J}(A)=\left\{\frac{[A x, x]}{[x, x]}: x \in \mathcal{H},[x, x] \neq 0\right\} .
$$

This is a generalization of the well-known classical numerical range of an operator in a Hilbert space

$$
W(A)=\left\{\frac{\langle A x, x\rangle}{\langle x, x\rangle}: x \in \mathcal{H},\langle x, x\rangle \neq 0\right\},
$$

a concept that has been investigated extensively in the last decades (e.g., see $[2,11,16])$, and plays an important role in the study of matrices and operators. There are many results which connect analytic and algebraic properties of an operator with the geometric properties of its numerical range. There is also a substantial interest in investigating these relations in the Krein space setting $([3,13,14])$.

A remarkable result of Ando [1] relating the numerical range with dilation theory, states the following:

Theorem 1.1. Let $A \in \mathcal{B}(\mathcal{H})$. The following conditions are equivalent.

(a) $W(A) \subseteq W(B)$ with $B=\left[\begin{array}{ll}0 & 2 \\ 0 & 0\end{array}\right]$.

(b) $A=V^{*}\left[\begin{array}{cc}0 & 2 I_{\mathcal{H}} \\ 0 & 0\end{array}\right] V$ for some $V$ satisfying $V^{*} V=I_{\mathcal{H}}$.

Since $\left[\begin{array}{cc}0 & 2 I_{\mathcal{H}} \\ 0 & 0\end{array}\right]=B \otimes I_{\mathcal{H}}$ with $B=\left[\begin{array}{ll}0 & 2 \\ 0 & 0\end{array}\right]$ and $W(B)=\mathcal{D}=\{z \in \mathbb{C}:|z| \leq 1\}$,

Theorem 1.1 (a) is equivalent to:

(c) $W(A)$ is included in the closed unit disc $\mathcal{D}$.

Ando's result can be easily rephrased in the context of dilation theory as follows. Given two linear operators $X$ and $Y$ acting on the Hilbert spaces $\mathcal{H}$ and $\mathcal{H}_{1}$, respectively, $Y$ is said to be a dilation of $X$ if there exists a linear operator $V: \mathcal{H} \rightarrow \mathcal{H}_{1}$ satisfying $V^{*} V=I_{\mathcal{H}}$ and $X=V^{*} Y V$. It is clear that if $A$ can be dilated to an operator of the form $B \otimes I_{\mathcal{H}}$, then $W(A) \subseteq W\left(B \otimes I_{\mathcal{H}}\right)=W(B)$. Thus, Ando's result states that if $B$ is a dilation of $A$, then $W(A) \subseteq W(B)$, and the converse also holds with $B=\left[\begin{array}{ll}0 & 2 \\ 0 & 0\end{array}\right]$.

Next we shift our attention to the case of Krein spaces. Let $X$ and $Y$ be linear operators acting in the Krein spaces $\mathcal{H}_{1}$ and $\mathcal{H}_{2}, \operatorname{dim} \mathcal{H}_{1}=n_{1}, \operatorname{dim} \mathcal{H}_{2}=n_{2}$, $n_{1}>n_{2}$, endowed with the indefinite metric matrices $J_{n_{1}}$ and $J_{n_{2}}$, respectively. We say that $Y: \mathcal{H}_{1} \rightarrow \mathcal{H}_{1}$ is a dilation of $X: \mathcal{H}_{2} \rightarrow \mathcal{H}_{2}$ if there exists a linear operator $V: \mathcal{H}_{2} \rightarrow \mathcal{H}_{1}$ such that

$$
V^{\#}=J_{n_{2}} V^{*} J_{n_{1}}, V^{\#} V=I_{n_{2}}, X=V^{\#} Y V,
$$

where $V^{\#}$ denotes the $J$-adjoint of $V$, defined as $[V x, y]=\left[x, V^{\#} y\right]$ for any $x \in \mathcal{H}_{1}, y \in \mathcal{H}_{2}$.

One of our aims is to study the relation between the inclusion $W(A) \subseteq W_{J}(B)$ and the condition that $\mathrm{A}$ can be dilated to an operator of the form $B \otimes I$. Our 
investigation should be viewed as a starting point to the research of the interface between the theory of dilations and the theory of Krein space numerical ranges. This program has been initiated in the classical case by Ando [1] and Mirman [17], and revisited by Choi and Li in [15].

This note is organized as follows. In Section 2, we collect results on the indefinite numerical range, directly related with our discussion. In Section 3, a criterion for hyperbolicity of $W_{J}(A)$ is given using a geometric approach. In Section 4, the hyperbolicity of the indefinite numerical range of generalized quadratic operators is stated. In Section 5, a characterization of $W_{J}(A)$ as a union of hyperbolical discs is obtained by a reduction to the two-dimensional case. In Section 6, Ando's result is extended to the setting of indefinite numerical ranges.

\section{PRELIMINARIES}

Let $J$ be an involutive Hermitian matrix $\left(J^{2}=J\right)$ with signature $(r, n-r)$, $0 \leq r \leq n$, that is, with $r$ positive eigenvalues and $n-r$ negative eigenvalues. In our discussion, we shall consider the positive and the negative J-numerical range:

$$
W_{J}^{ \pm}(A)=\left\{[A x, x]: x \in \mathbb{C}^{n},[x, x]= \pm 1\right\} .
$$

It can be easily seen that

$$
W_{J}(A)=W_{J}^{+}(A) \cup W_{J}^{-}(A),
$$

and

$$
W_{-J}^{+}(A)=-W_{J}^{-}(A) .
$$

So, we may concentrate in the investigation of one of these sets. In [10] it has been proved that $W_{J}(A)$ is a pseudo-convex subset of $\mathbb{C}$, i.e., for any distinct pair of points $x, y \in W_{J}(A)$, either the line segment $[x, y]$ or the half-rays $\alpha x+(1-\alpha) y$, $\alpha \leq 0$ and $\alpha \geq 1$ are contained in $W_{J}(A)$. In contrast with the classical case, $W_{J}(A)$ may be neither closed nor bounded, even in the finite dimensional case (see, [12, Section 2]). In general, it is difficult to obtain the complete characterization of the Krein space numerical range. However, in the case of operators acting on a two-dimensional space this description is simple [6, Theorem 3.2], and usually known as the Hyperbolical Range Theorem. In the sequel, we denote by $J_{2}$ the matrix diag $(1,-1)$.

Theorem 2.1. If $A \in M_{2}$ has eigenvalues $\lambda_{1}$ and $\lambda_{2}$, then $W_{J_{2}}(A)$ is a possibly degenerate two-component hyperbolical disc with foci at $\lambda_{1}$ and $\lambda_{2}$, transverse and nontransverse axis of length

$$
\sqrt{\operatorname{Tr}\left(A^{\#} A\right)-2 \operatorname{Re}\left(\lambda_{1} \bar{\lambda}_{2}\right)} \text { and } \sqrt{\left|\lambda_{1}\right|^{2}+\left|\lambda_{2}\right|^{2}-\operatorname{Tr}\left(A^{\#} A\right)}
$$

, respectively. For the degenerate cases, $W_{J_{2}}(A)$ may be a singleton, a line, the union of two half-lines, the whole complex plane, or the complex plane except a line.

The following properties will be frequently used in the sequel:

(P1) $W_{J}(\alpha A+\beta B)=\alpha W_{J}(A)+\beta W_{J}(B)$ for any $A, B \in M_{n}, \alpha, \beta \in \mathbb{C}$.

(P2) Given $A \in M_{n}, W_{J}\left(U^{\#} A U\right)=W_{J}(A)$ for any $J$-unitary matrix $U$, that is, $U \in M_{n}$ satisfying $U^{\#} U=I$. 


\section{Hyperbolicity of $W_{J}(A)$}

It has been shown recently that the Krein space numerical range of some matrices and operators is a two-component hyperbolical disc. This is the case of certain tridiagonal operators [4] and of quadratic operators, i.e., those with minimal polynomial of degree 2 [5]. In this section, we present a necessary and sufficient condition for hyperbolicity of the Krein space numerical range.

For this purpose, we recall that the support function $p_{E}$ of a convex subset of the complex plane $E$, is defined as

$$
p_{E}(\theta)=\sup \left\{\operatorname{Re}\left(\mathrm{e}^{-i \theta} z\right): z \in E\right\},
$$

for $\theta \in[0,2 \pi]$. According to the definition, the value $p_{E}(\theta)$ is the maximum scalar projection of the set $E$ in the direction of $\theta$. If $E$ contains the origin, we clearly have $p_{E}(\theta) \geq 0$, for all $\theta$. If a line $L$ is extended from the origin in the direction of the vector $(\cos \theta, \sin \theta)$, then $p_{E}(\theta)$ is the distance from the origin to a line $L^{\prime}$ perpendicular to $L$ which is tangent to $\partial E$, the boundary of $E$, at some point. The support function completely determines the closure of $E$, in the sense that if $p_{E}(\theta)=p_{F}(\theta)$ for convex sets $E$ and $F$ and all real $\theta$, then $\bar{E}=\bar{F}$. When the set $E$ is an ellipse, the support function has a simple formula. This result can be found in several standard references about convex sets as well as in [9]. Next we extend this concept to $W_{J}(A)$. To avoid cases of degeneracy in which $W_{J}^{ \pm}(A)$ is the whole plane, a half-plane, a line or a half-line, we assume that there exists a real interval $\left[\theta_{1}, \theta_{2}\right]$ such that when $\theta$ ranges over it either (i) $\operatorname{Re} W_{J}^{-}\left(\mathrm{e}^{-i \theta} A\right)<\operatorname{Re} W_{J}^{+}\left(\mathrm{e}^{-i \theta} A\right)$ or (ii) $\operatorname{Re} W_{J}^{-}\left(\mathrm{e}^{-i \theta} A\right)>\operatorname{Re} W_{J}^{+}\left(\mathrm{e}^{-i \theta} A\right)$. For $\theta \in\left[\theta_{1}, \theta_{2}\right]$ and assuming that (i) holds, the support functions of $W_{J}^{ \pm}(A)$ are defined as

$$
p_{W^{-}}(\theta):=\sup \left\{\operatorname{Re}\left(\mathrm{e}^{-i \theta}[A x, x]\right): x \in W_{J}^{-}(A)\right\}
$$

and

$$
p_{W^{+}}(\theta):=\inf \left\{\operatorname{Re}\left(\mathrm{e}^{-i \theta}[A x, x]\right): x \in W_{J}^{+}(A)\right\} .
$$

For $\theta \in\left[\theta_{1}, \theta_{2}\right]$ such that $\operatorname{Re} W_{J}^{-}\left(\mathrm{e}^{-i \theta} A\right)>\operatorname{Re} W_{J}^{+}\left(\mathrm{e}^{-i \theta} A\right)$, we define

$$
p_{W^{-}}(\theta):=\inf \left\{\operatorname{Re}\left(\mathrm{e}^{-i \theta}[A x, x]\right): x \in W_{J}^{-}(A)\right\}
$$

and

$$
p_{W^{+}}(\theta):=\sup \left\{\operatorname{Re}\left(\mathrm{e}^{-i \theta}[A x, x]\right): x \in W_{J}^{+}(A)\right\} .
$$

Considering the matrix

$$
H_{\theta}=\operatorname{Re}\left(\mathrm{e}^{-i \theta} A\right):=\frac{\mathrm{e}^{-i \theta} A+\mathrm{e}^{-i \theta} A^{\#}}{2},
$$

which is $J$-Hermitian or ( $J$-self-adjoint) since $H_{\theta}^{\#}=H_{\theta}$, then, we can alternatively define the support functions of $W_{J}(A)$ as follows:

$$
p_{W^{-}}(\theta)=\sup \left\{W_{J}^{-}\left(H_{\theta}\right): \quad \theta \in\left[\theta_{1}, \theta_{2}\right]\right\}
$$

and

$$
p_{W^{+}}(\theta)=\inf \left\{W_{J}^{+}\left(H_{\theta}\right): \quad \theta \in\left[\theta_{1}, \theta_{2}\right]\right\} .
$$

Next we characterize the support functions of $W_{J}^{ \pm}(A)$, when these sets are the components of a 2-component hyperbolical disc $H$. Without loss of generality, we 
can always assume $H$ centered at the origin and with axis along the coordinate axis, by rotating and translating $A$, as the $J$-numerical range satisfies (P1) and (P2).

Theorem 3.1. Let $a, b>0$ and $A \in M_{n}$. Then $W_{J}(A)$ is the hyperbolical disc $H$ with equation $\frac{x^{2}}{a^{2}}-\frac{y^{2}}{b^{2}} \geq 1$ if and only if

$$
p_{W^{-}}(\theta)=-\sqrt{a^{2} \cos ^{2} \theta-b^{2} \sin ^{2} \theta},
$$

and

$$
p_{W^{+}}(\theta)=\sqrt{a^{2} \cos ^{2} \theta-b^{2} \sin ^{2} \theta}
$$

for $\theta \in\left[\theta_{1}, \theta_{2}\right]$.

Proof. We prove the direct implication. Consider the unit vector $(\cos \theta, \sin \theta)$ in the direction of $\theta$. The equation of the supporting line perpendicular to this vector and at a distance $\lambda$ from the origin is

$$
x \cos \theta+y \sin \theta=\lambda .
$$

We denote by $H_{+}\left(H_{-}\right)$the branch of the hyperbolical disc $H$ in the right halfplane (left half-plane). The slope of the tangent to $H_{+}$at $(x, y)$ is given by

$$
\frac{x}{a^{2}}-\frac{y}{b^{2}} \frac{d y}{d x}=0 \text {. }
$$

Thus,

$$
y^{\prime}=\frac{x b^{2}}{y a^{2}}=-\cot \theta
$$

which implies that

$$
\frac{x}{a^{2} \cos \theta}=-\frac{y}{b^{2} \sin \theta} .
$$

Squaring and performing some elementary computations, it follows that

$$
\frac{\frac{x^{2}}{a^{2}}}{a^{2} \cos ^{2} \theta}=\frac{\frac{y^{2}}{b^{2}}}{b^{2} \sin ^{2} \theta}=\frac{\frac{x^{2}}{a^{2}}-\frac{y^{2}}{b^{2}}}{a^{2} \cos ^{2} \theta-b^{2} \sin ^{2} \theta}=\frac{1}{a^{2} \cos ^{2} \theta-b^{2} \sin ^{2} \theta} .
$$

From (3.1) we easily get

$$
\begin{aligned}
\frac{x}{a^{2} \cos \theta} & =-\frac{y}{b^{2} \sin \theta}=\frac{1}{\sqrt{a^{2} \cos ^{2} \theta-b^{2} \sin ^{2} \theta}} \\
& =\frac{x \cos \theta+y \sin \theta}{a^{2} \cos ^{2} \theta-b^{2} \sin ^{2} \theta} \\
& =\frac{\lambda}{a^{2} \cos ^{2} \theta-b^{2} \sin ^{2} \theta} .
\end{aligned}
$$

So

$$
\lambda=\sqrt{a^{2} \cos ^{2} \theta-b^{2} \sin ^{2} \theta} .
$$


Using analogous arguments for $H_{-}$, the direct implication follows.

Now we prove that the converse holds. The supporting line of $W_{J}^{+}(A)$ in the direction perpendicular to $\theta$, and at the distance $\sqrt{a^{2} \cos ^{2} \theta-b^{2} \sin ^{2} \theta}$ from the origin, is

$$
x \cos \theta+y \sin \theta=\sqrt{a^{2} \cos ^{2} \theta-b^{2} \sin ^{2} \theta} .
$$

We compute the envelope of the family of supporting lines of $W_{J}^{+}(A)$, which describes its boundary. For this purpose, we consider the following system of linear equations in $x, y$

$$
x \cos \theta+y \sin \theta=\sqrt{a^{2} \cos ^{2} \theta-b^{2} \sin ^{2} \theta}
$$

and

$$
-x \sin \theta+y \cos \theta=\frac{-a^{2} \cos \theta \sin \theta-b^{2} \sin \theta \cos \theta}{\sqrt{a^{2} \cos ^{2} \theta-b^{2} \sin ^{2} \theta}} .
$$

Its solution is given by

$$
x=\frac{a^{2} \cos \theta}{\sqrt{a^{2} \cos ^{2} \theta-b^{2} \sin ^{2} \theta}} \quad \text { and } \quad y=-\frac{b^{2} \sin \theta}{\sqrt{a^{2} \cos ^{2} \theta-b^{2} \sin ^{2} \theta}} .
$$

So

$$
\frac{x^{2}}{a^{2}}-\frac{y^{2}}{b^{2}}=\frac{a^{2} \cos ^{2} \theta-b^{2} \sin ^{2} \theta}{a^{2} \cos ^{2} \theta-b^{2} \sin ^{2} \theta}=1 .
$$

The envelope of the family of supporting lines of $W_{J}^{-}(A)$ is similarly obtained.

\section{KREIN Numerical RANGES OF GENERALIZED QUADRATIC OPERATORS}

Consider an operator $A \in B(\mathcal{H})$ with an operator matrix of the form

$$
A=\left[\begin{array}{cc}
a I_{r} & c P \\
d P & -a I_{r}
\end{array}\right], \quad a \in \mathbb{R}, \quad c, d \in \mathbb{C},
$$

where $P$ is a positive semi-definite matrix and $c d P \neq 0$. If $d=0$, such an operator reduces to a so-called quadratic operator (see [5] and the references therein).

The family of matrices

$$
A_{p}=\left[\begin{array}{cc}
a & c p \\
d p & -a
\end{array}\right], \quad a \in \mathbb{R}, \quad p \geq 0
$$

will be very useful in our investigation. The following lemma is used in the proof of Theorem 4.2 .

Lemma 4.1. Let $A_{p}$ be of the form (4.2). For $p \geq 0, W_{J_{2}}\left(A_{p}\right)$ is the pseudoconvex region bounded by the two component hyperbola with foci at

$$
\mu_{p}^{ \pm}= \pm \sqrt{a^{2}+c d p^{2}}
$$

and transverse axis of length

$$
\sqrt{2\left|a^{2}+c d p^{2}\right|-\left(2 a^{2}+\left(|c|^{2}+|d|^{2}\right) p^{2}\right)} .
$$

If $p<q$, then $W_{J_{2}}\left(A_{p}\right) \subseteq W_{J_{2}}\left(A_{q}\right)$. Further, 
(1) If $c=\bar{d}$, then $W_{J_{2}}\left(A_{p}\right)$ is the union of two half-rays whose endpoints are $\mu_{p}^{+}$and $\mu_{p}^{-}$, and such that $W_{J_{2}}\left(A_{p}\right)$ is a subset of the relative interior of $W_{J_{2}}\left(A_{q}\right)$.

(2) If $c \neq \bar{d}$ and $|c|=|d|$, then $\partial W_{J_{2}}\left(A_{p}\right) \cap W_{J_{2}}\left(A_{q}\right)=\{a,-a\}$ and $W_{J_{2}}\left(A_{p}\right) \subseteq$ $\operatorname{int}\left(W_{J_{2}}\left(A_{q}\right)\right) \cup\{a,-a\}$.

(3) If $|c| \neq|d|$, then $W_{J_{2}}\left(A_{p}\right) \subseteq \operatorname{int} W_{J_{2}}\left(A_{q}\right)$.

Proof. The $J$-Hermitian part of $\mathrm{e}^{-i \theta} A_{p}$ reads

$$
\begin{aligned}
& \operatorname{Re}\left(\mathrm{e}^{-i \theta} A_{p}\right)=H_{p}^{\theta}=\frac{\left(\mathrm{e}^{-i \theta} A_{p}+\mathrm{e}^{i \theta} J_{2} A_{p}^{*} J_{2}\right)}{2} \\
& =\left[\begin{array}{cc}
a \cos \theta & \frac{1}{2}((c-\bar{d}) \cos \theta-i(c+\bar{d}) \sin \theta) p \\
\frac{1}{2}((-\bar{c}+d) \cos \theta-i(\bar{c}+d) \sin \theta) p & -a \cos \theta
\end{array}\right],
\end{aligned}
$$

and its eigenvalues are given by

$$
\lambda^{ \pm}\left(H_{p}^{\theta}\right)= \pm \frac{1}{2} \sqrt{\left(2 a^{2}-\left(|c|^{2}+|d|^{2}\right) p^{2}+\left(2 a^{2}+(c d+\bar{c} \bar{d}) p^{2}\right) \cos (2 \theta)-i(c d-\bar{c} \bar{d}) p^{2}\right) \sin (2 \theta)} .
$$

For $\theta \in\left[\theta_{1}, \theta_{2}\right]$ such that

$$
\left(2 a^{2}-\left(|c|^{2}+|d|^{2}\right) p^{2}+\left(2 a^{2}+(c d+\bar{c} \bar{d}) p^{2}\right) \cos (2 \theta)-i(c d-\bar{c} \bar{d}) p^{2}\right) \sin (2 \theta) \geq 0,
$$

it follows that

$$
\left(\lambda^{ \pm}\left(H_{p}^{\theta}\right)\right)^{2} \leq\left(\lambda^{ \pm}\left(H_{p}^{\theta}\right)\right)^{2}
$$

for $p<q$, because $\lambda^{+}\left(H_{p}^{\theta}\right)$ is an increasing function of $\theta$.

1. It can be readily seen that the equality occurs in the above inequality if and only if $|c|=|d|$ and $\theta$ is such that $\left(2 a^{2}-\left(|c|^{2}+|d|^{2}\right) p^{2}+\left(2 a^{2}+(c d+\right.\right.$ $\left.\left.\bar{c} \bar{d}) p^{2}\right) \cos (2 \theta)-i(c d-\bar{c} \bar{d}) p^{2}\right) \sin (2 \theta)=0$. The result follows from the previous observations, bearing in mind the Hyperbolical Range Theorem.

2. If $|c|=|d|$, then the above mentioned equality case does not depend on $p$. This implies that there exist common points to all the hyperbolical discs boundaries. Further, these points are $-a$ and $a$.

3. Clear.

Given a self-adjoint operator $X \in B(\mathcal{H})$, diagonalizable under a $J$-unitary similarity, define

$$
\sigma_{J}^{ \pm}(X)=\{\lambda \in \mathbb{R}: X \xi=\lambda \xi, \text { for some } \xi \in \mathcal{H} \text { with }[\xi, \xi]= \pm 1\} .
$$

Throughout, $\|X\|$ denotes the largest eigenvalue of $X$.

Theorem 4.2. Suppose $A \in B(\mathcal{H})$ has an operator matrix of the form (4.1). Let $J=I_{r} \oplus-I_{r}, \tilde{p}=\|P\|$ and

$$
A_{\tilde{p}}=\left[\begin{array}{cc}
a & c \tilde{p} \\
d \tilde{p} & -a
\end{array}\right]
$$

so that $A_{\tilde{p}}$ has eigenvalues $\mu_{\tilde{p}}^{ \pm}= \pm \sqrt{a^{2}+c d \tilde{p}^{2}}$, and $W_{J_{2}}\left(A_{\tilde{p}}\right)$ is the pseudo-convex region bounded by the 2-component hyperbola with foci at $\mu_{\tilde{p}}^{+}, \mu_{\tilde{p}}^{-}$, and transverse axis of length

$$
\sqrt{2\left|a^{2}+c d \tilde{p}^{2}\right|-\left(2 a^{2}+\left(|c|^{2}+|d|^{2}\right) \tilde{p}^{2}\right)} \text {. }
$$


Then,

$$
W_{J}(A)=W_{J_{2}}\left(A_{\tilde{p}}\right)
$$

Proof. We notice that according to the hypothesis, $A$ is $J$-unitarily similar to

$$
\tilde{A}=\left[\begin{array}{cc}
a I_{r} & c \tilde{P} \\
d \tilde{P} & -a I_{r}
\end{array}\right]
$$

where $\tilde{P}=\operatorname{diag}\left(p_{1}, p_{2}, \ldots, p_{r}\right)$, with $p_{1} \geq p_{2} \geq \ldots \geq p_{r}$ the eigenvalues of $\tilde{P}$.

For $\theta \in\left[\theta_{1}, \theta_{2}\right]$, consider the $J$-self-adjoint matrix $H^{\theta}=\operatorname{Re}\left(\mathrm{e}^{-i \theta} \tilde{A}\right)$. By Lemma 4.1 , it follows that

$$
\sigma_{J}^{+}\left(H^{\theta}\right)=\bigcup_{j=1}^{r} \sigma_{J}^{+}\left(H_{p_{j}}^{\theta}\right)
$$

and

$$
\sigma_{J}^{-}\left(H^{\theta}\right)=\bigcup_{j=1}^{r} \sigma_{J}^{-}\left(H_{p_{j}}^{\theta}\right)
$$

Denote by $\lambda_{J M}^{-}(X)$ the largest eigenvalue of the $J$-self-adjoint matrix $X \in B(\mathcal{H})$ in $\sigma_{J}^{-}(X)$, and by $\lambda_{J m}^{+}(X)$ the smallest eigenvalue of $X$ in $\sigma_{J}^{+}(X)$. Since $\lambda_{J M}^{-}\left(H^{\theta}\right)$ is the eigenvalue in $\sigma_{J_{2}}^{-}\left(\mathrm{e}^{-i \theta} \tilde{A}\right)$ and $\lambda_{J m}^{+}\left(H^{\theta}\right)$ is the eigenvalue in $\sigma_{J_{2}}^{+}\left(\mathrm{e}^{-i \theta} \tilde{A}\right)$, the result easily follows.

\section{Compressions}

Let $P \in M_{2}$ be a $J_{2}$-orthogonal projection, i.e., $P^{2}=P, P^{\#}=P$. For $A \in M_{n}$, the restriction of $P A P$ to the range of $P$ is called a 2-dimensional compression of $A$, or in matrix form

$$
A_{x y}=\left[\begin{array}{cc}
\epsilon_{x}[A x, x] & \epsilon_{x}[A y, x] \\
\epsilon_{y}[A x, y] & \epsilon_{y}[A y, y]
\end{array}\right]
$$

where $x$ e $y$ are real $J$-orthonormal column $n$-tuples, i.e.,

$$
[x, y]=0, \quad \epsilon_{x}=[x, x]= \pm 1 \quad \text { and } \quad \epsilon_{y}=[y, y]= \pm 1 .
$$

Explicitly, we have $P A P=A_{x y} \oplus 0_{n-2}$, the zero block of size $n-2$.

Theorem 5.1. Let $A \in M_{n}$ and $J=I_{r} \oplus\left(-I_{n-r}\right)$. Then $W_{J}(A)$ is the union of all the sets

$$
\left(\bigcup_{\substack{x, y \in \mathbb{R}^{n} \\[x, x]=[y, y]=1}} W_{J_{x y}}\left(A_{x y}\right)\right) \bigcup\left(\bigcup_{\substack{x, y \in \mathbb{R}^{n} \\[x, x]=[y, y]=-1}} W_{J_{x y}}\left(A_{x y}\right)\right) \bigcup\left(\bigcup_{\substack{x, y \in \mathbb{R}^{n} \\[x, x]=-[y, y]=1}} W_{J_{x y}}\left(A_{x y}\right)\right),
$$

where $A_{x y}$ is the matrix (5.1), $x$ and $y$ run over all pairs of real $J$-orthonormal vectors and $J_{x y}=\operatorname{diag}\left(\epsilon_{x}, \epsilon_{y}\right)$, with $\epsilon_{x}$ and $\epsilon_{y}$ given by (5.2).

Proof. Let $w=u+i v$ be a complex vector in which $u$ and $v$ are real $n$-vectors. Assume that $[w, w]=1$, and so $[A w, w] \in W_{J}^{+}(A)$ (the case $[A w, w] \in-W_{J}^{-}(A)$ is similarly treated). Thus,

$$
1=[w, w]=[u, u]+[v, v] .
$$


If $u$ and $v$ are linearly dependent, say $v=\alpha u, \alpha \in \mathbb{R}$, then $w=u+i \alpha u=(1+i \alpha) u$ and hence

$$
[A w, w]=[A|1+i \alpha| u,|1+i \alpha| u] .
$$

Since $|1+i \alpha| u$ is a real $J$-unit vector, from (5.4) we can conclude that $[A w, w] \in$ $W_{J_{2}}^{+}\left(A_{x y}\right)$, where $x=|1+i \alpha| u$ and $y$ is chosen to be a real $J$-unit vector such that $[x, y]=0$.

Assume that $u$ and $v$ are linearly independent. We have $[w, w]=[u, u]+[v, v] \neq$ 0 . Let $[u, u] \neq 0$, and

$$
x=\frac{u}{\sqrt{|[u, u]|}}
$$

Take

$$
s=[u, v] u-[u, u] v,
$$

so that $[s, u]=0$. Assume that $[s, s]=[u, u][u, v]([u, u]-[u, v]) \neq 0$ and let

$$
y=\frac{s}{\sqrt{|[s, s]|}} \text {. }
$$

We may write

$$
w=u+i v=\alpha_{x} x+\alpha_{y} y
$$

where

$$
\alpha_{x}=\sqrt{|[u, u]|}\left(1+i \frac{[u, v]}{[u, u]}\right), \quad \alpha_{y}=-i \frac{\sqrt{|[s, s]|}}{[u, u]} .
$$

Let us compute $[A w, w]$. We find

$$
\begin{aligned}
& {[A w, w]=\alpha_{x} \bar{\alpha}_{x}[A x, x]+\alpha_{y} \bar{\alpha}_{y}[A y, y]+\alpha_{x} \bar{\alpha}_{y}[A x, y]+\alpha_{y} \bar{\alpha}_{x}[A y, x]} \\
& =\left[\bar{\alpha}_{x}, \bar{\alpha}_{y}\right]\left[\begin{array}{cc}
\epsilon_{x} & 0 \\
0 & \epsilon_{y}
\end{array}\right]\left[\begin{array}{ll}
{[A x, x] \epsilon_{x}} & {[A x, y] \epsilon_{x}} \\
{[A y, x] \epsilon_{y}} & {[A y, y] \epsilon_{y}}
\end{array}\right]\left[\begin{array}{c}
\alpha_{x} \\
\alpha_{y}
\end{array}\right] \\
& {[w, w]=\alpha_{x} \bar{\alpha}_{x}[x, x]+\alpha_{y} \bar{\alpha}_{y}[y, y]=\left[\bar{\alpha}_{x}, \bar{\alpha}_{y}\right]\left[\begin{array}{cc}
\epsilon_{x} & 0 \\
0 & \epsilon_{y}
\end{array}\right]\left[\begin{array}{c}
\alpha_{x} \\
\alpha_{y}
\end{array}\right]}
\end{aligned}
$$

so that

$$
\frac{[A w, w]}{[w, w]}=\frac{\left[A_{x y} z, z\right]}{[z, z]},
$$

where $z=\left[\alpha_{x}, \alpha_{y}\right]^{T}$. The equality (5.5) shows that any element $[A w, w]$ in $W_{J}^{+}(A)$ is in the $J_{2}$-numerical range of some 2 -dimensional real $J$-orthogonal compression of $A$.

If $[s, s]=0$, we perturb $w$ so that $[s, s] \neq 0$. For this purpose, we consider $w^{\prime}=u^{\prime}+i v^{\prime}, u^{\prime}, v^{\prime} \in \mathbb{R}$, such that $\left[u^{\prime}, u^{\prime}\right]\left[u^{\prime}, v^{\prime}\right]\left(\left[u^{\prime}, u^{\prime}\right]-\left[u^{\prime}, v^{\prime}\right]\right) \neq 0$ and replace $w$ by $w_{\epsilon}=\epsilon w+(1-\epsilon) w^{\prime}$. For a sufficiently small $\epsilon$, we have

$$
\left[u_{\epsilon}, u_{\epsilon}\right]\left[u_{\epsilon}, v_{\epsilon}\right]\left(\left[u_{\epsilon}, u_{\epsilon}\right]-\left[u_{\epsilon}, v_{\epsilon}\right]\right) \neq 0 .
$$

Further, the point generated by $w_{\epsilon}$ is in the neighborhood of the point generated by $w$, and approaches it as $\epsilon \rightarrow 0$.

The reciprocal inclusion is a consequence of the following facts. Any 2-dimensional real $J$-orthogonal compression of $A$ is a 2 -square principal submatrix of a matrix 
$J$-orthogonally similar to $A$, and $W_{J}(A)$ is invariant under $J$-orthogonal similarities. Moreover, $W_{J^{\prime}}(B) \subset W_{J}(A)$ for any principal submatrix $B$ of $A$ and $J^{\prime}$ the principal submatrix of $J$ in the same rows and columns.

The pseudo-convexity of $W_{J}(A)$ is a simple consequence of the previous theorem. It was obtained in [12] by a different approach.

Corollary 5.2. Let $A \in M_{n}$ and $J=I_{r} \oplus\left(-I_{n-r}\right)$. Then $W_{J}^{ \pm}(A)$ are convex sets and $W_{J}(A)$ is a pseudo-convex subset of $\mathbb{C}$.

Proof. Consider the points $[A u, u] /[u, u],[A w, w] /[w, w] \in W_{J}(A)$. Let $s=[w, u] u-$ $[u, u] w$. Assume that $[s, s] \neq 0$. The vectors $x=u / \sqrt{|[u, u]|}$ and $y=s / \sqrt{|[s, s]|}$ are $J$-orthonormal. If $\epsilon_{x} \epsilon_{y}>0$, the numerical range of the compression of $A$ to the subspace spanned by the vectors $x, y$ is an elliptical disc with foci at the eigenvalues $\lambda_{1}, \lambda_{2}$ of $A_{x y}$ and minor axis of length ( $\left.\operatorname{Tr} A A^{*}-\left|\lambda_{1}\right|^{2}-\left|\lambda_{2}\right|^{2}\right)^{1 / 2}[11$, Theorem 1.3.6]. If $\epsilon_{x} \epsilon_{y}<0$, the mentioned numerical range is a hyperbolical disc containing the points $[A u, u] /[u, u]$ and $[A w, w] /[w, w]$.

If $[s, s]=0$, we perturb $s$ as $s_{\epsilon}=s+t \epsilon$, where $[t, t] \neq 0$, and $[u, t]=0$, so that $\left[s_{\epsilon}, s_{\epsilon}\right] \neq 0$ for a sufficiently small $\epsilon$. Further, the points generated by the vectors $u, w_{\epsilon}=\left(s_{\epsilon}-[w, u] u\right) /[u, u]$ are in the neighborhoods of the points generated by $u, w$, respectively, and approach them as $\epsilon \rightarrow 0$.

In the next theorem, the numerical range is compressed into the union of 2-by-2 compressions over certain $J$-orthogonally complementary subspaces.

Theorem 5.3. Let $A \in M_{n}, J=I_{r} \oplus\left(-I_{n-r}\right)$ and $S$ be a nondegenerate subspace of $\mathbb{C}^{n}$. Then

$$
W_{J}(A)=\left(\bigcup_{x, y} W_{J_{2}}\left(A_{x y}\right)\right)
$$

where $x$ and $y$ vary over all $J$-unit vectors in $S$ and $S^{\perp}$, respectively.

Proof. For any $J_{2}$-unit vector $(\alpha, \beta)^{T} \in \mathbb{C}^{2}$, we have $[\alpha x+\beta y, \alpha x+\beta y]= \pm 1$. Then for $w=\alpha x+\beta y$ we obtain

$$
\begin{aligned}
& {[A w, w]=\alpha \bar{\alpha}[A x, x]+\beta \bar{\beta}[A y, y]+\alpha \bar{\beta}[A x, y]+\beta \bar{\alpha}[A y, x]} \\
& =[\bar{\alpha}, \bar{\beta}]\left[\begin{array}{cr}
\epsilon_{x} & 0 \\
0 & \epsilon_{y}
\end{array}\right]\left[\begin{array}{ll}
{[A x, x] \epsilon_{x}} & {[A x, y] \epsilon_{x}} \\
{[A y, x] \epsilon_{y}} & {[A y, y] \epsilon_{y}}
\end{array}\right]\left[\begin{array}{c}
\alpha \\
\beta
\end{array}\right] \\
& {[w, w]=\alpha \bar{\alpha}[x, x]+\beta \bar{\beta}[y, y]=[\bar{\alpha}, \bar{\beta}]\left[\begin{array}{cc}
\epsilon_{x} & 0 \\
0 & \epsilon_{y}
\end{array}\right]\left[\begin{array}{c}
\alpha \\
\beta
\end{array}\right]}
\end{aligned}
$$

so that

$$
W_{J_{2}}\left(A_{x y}\right) \subset W_{J}(A) .
$$

Conversely, if $z \in \mathbb{C}^{n}$ is an arbitrary $J$-unit vector, then there exist $J$-unit vectors $x \in S, y \in S^{\perp}$ and a $J_{2}$-unit vector $(\alpha, \beta)^{T} \in \mathbb{C}^{2}$ such that $z=\alpha x+\beta y$. It can be easily shown that $[A z, z] \in W_{J_{2}}\left(A_{x y}\right)$, and the result follows. 
Remark 5.4. In [13] and [8] algorithms for plotting the indefinite numerical range have been presented. Theorem 5.1 may be used to provide an alternative algorithm for the numerical determination of $W_{J}(A)$. The idea is to generate a complete set of $J$-orthonormal pairs $x, y$ and actually compute the union of the hyperbolical and elliptical discs $W_{J}\left(A_{x y}\right)$.

\section{Dilations}

Consider the Krein spaces $\mathcal{H}_{1}, \mathcal{H}_{2}$ and $\mathcal{H}_{3}=\mathcal{H}_{1} \otimes \mathcal{H}_{2}$, with $n_{1}=2, n_{2}=n$, and the involutions $J_{2}=\operatorname{diag}(1,-1)$ and $J_{2} \otimes I_{n}=J_{2 n}$ acting on $\mathcal{H}_{1}$ and $\mathcal{H}_{2}$, respectively. Let

$$
B=\left[\begin{array}{cc}
\lambda_{1} & d \\
0 & \lambda_{2}
\end{array}\right]
$$

Since

$$
B \otimes I_{n}=\left[\begin{array}{cc}
\lambda_{1} I_{n} & d I_{n} \\
0 & \lambda_{2} I_{n}
\end{array}\right]=\bigoplus_{1}^{n}\left[\begin{array}{cc}
\lambda_{1} & d \\
0 & \lambda_{2}
\end{array}\right]
$$

we conclude that

$$
W_{J_{2 n}}\left(B \otimes I_{n}\right)=W_{J_{2}}(B) .
$$

Let $u_{1}, \cdots u_{2 n}$ be a $J_{2 n}$-orthonormal basis for $\mathcal{H}_{3}$, and let $V$ be the $2 n \times n$ matrix $V=\left(u_{1}, \cdots, u_{n}\right)$ whose $j$ th column is $u_{j}$. If $\left[u_{1}, u_{1}\right]=\cdots=\left[u_{n}, u_{n}\right]=1$, we obtain

$$
V^{*} J_{2 n} V=I_{n}
$$

Consider

$$
A=V^{*}\left(J_{2} \otimes I_{n}\right)\left(B \otimes I_{n}\right) V .
$$

There is a simple inclusion relation between $W(A)$ and $W_{J_{2}}(B)$. Indeed, for $x \in$ $\mathcal{H}_{n}$ we find

$$
\frac{x^{*} A x}{x^{*} x}=\frac{x^{*} V^{*}\left(J_{2} \otimes I_{n}\right)\left(B \otimes I_{n}\right) V x}{x^{*} V^{*}\left(J_{2} \otimes I_{n}\right) V x}=\frac{y^{*}\left(J_{2} \otimes I_{n}\right)\left(B \otimes I_{n}\right) y}{y^{*}\left(J_{2} \otimes I_{n}\right) y},
$$

where $y=V u$. Thus,

$$
W(A) \subseteq W_{J_{2}}(B)
$$

Next we consider the compression of $B \otimes I_{n}$ to the space spanned by $u_{\sigma_{1}}, \cdots, u_{\sigma_{n}}$, with

$$
\left[u_{\sigma_{1}}, u_{\sigma_{1}}\right]=\epsilon_{\sigma_{1}}, \ldots,\left[u_{\sigma_{n}}, u_{\sigma_{n}}\right]=\epsilon_{\sigma_{n}}
$$

$\sigma \in S_{2 n}$, the symmetric group of degree $2 n$. If we take the $2 n \times n$ matrix $V=$ $\left(u_{\sigma_{1}}, \cdots, u_{\sigma_{n}}\right)$, we have

$$
V^{*} J_{2 n} V=V^{*}\left(J_{2} \otimes I_{n}\right) V=J_{n}^{\sigma}=\operatorname{diag}\left(\epsilon_{\sigma_{1}}, \cdots \epsilon_{\sigma_{n}}\right) .
$$

Consider now

$$
A=J_{n}^{\sigma} V^{*}\left(J_{2} \otimes I_{n}\right)\left(B \otimes I_{n}\right) V .
$$

The following inclusion holds

$$
W_{J_{n}^{\sigma}}^{ \pm}(A) \subseteq W_{J_{2}}^{ \pm}(B)
$$


because, for $x \in \mathcal{H}_{n}$ and $y=U v$, we get

$$
\frac{x^{*} J_{n}^{\sigma} A x}{x^{*} J_{n}^{\sigma} x}=\frac{x^{*} V^{*}\left(J_{2} \otimes I_{n}\right)\left(B \otimes I_{n}\right) V x}{x^{*} V^{*}\left(J_{2} \otimes I_{n}\right) V x}=\frac{y^{*}\left(J_{2} \otimes I_{n}\right)\left(B \otimes I_{n}\right) y}{y^{*}\left(J_{2} \otimes I_{n}\right) y} .
$$

Theorem 6.1. Let

$$
B=\operatorname{diag}\left(\lambda_{1}, \lambda_{2}\right), \lambda_{1}>\lambda_{2} .
$$

If A satisfies $W(A) \subseteq W_{J_{2}}^{+}(B)$ or $W(A) \subseteq W_{J_{2}}^{-}(B)$, then $A$ can be dilated to an operator of the form $B \otimes I_{n}$.

Proof. We have

$$
B \otimes I_{n}=\left[\begin{array}{cc}
\lambda_{1} I_{n} & 0 \\
0 & \lambda_{2} I_{n}
\end{array}\right], \quad J_{2} \otimes I_{n}=\left[\begin{array}{cc}
I_{n} & 0 \\
0 & -I_{n}
\end{array}\right] .
$$

Let us first assume that $W(A) \subseteq W_{J_{2}}^{+}(B)$. By the Hyperbolical Range Theorem we may conclude that $W_{J_{2}}^{+}(B)=\left[\lambda_{1},+\infty[\right.$. As a consequence, $A$ is Hermitian since $W(A) \subseteq\left[\lambda_{1},+\infty\left[\right.\right.$, and so $\left(A-\lambda_{1} I_{n}\right),\left(A-\lambda_{2} I_{n}\right)$ are positive semidefinite matrices.

Let us define

Since

$$
C=\sqrt{\frac{A-\lambda_{2} I_{n}}{\lambda_{1}-\lambda_{2}}}, \quad D=\sqrt{\frac{A-\lambda_{1} I_{n}}{\lambda_{1}-\lambda_{2}}} .
$$

$$
\left[\begin{array}{ll}
C & D
\end{array}\right]\left(J_{2} \otimes I_{n}\right)\left[\begin{array}{l}
C \\
D
\end{array}\right]=\left[\begin{array}{ll}
C & D
\end{array}\right]\left[\begin{array}{cc}
I_{n} & 0 \\
0 & -I_{n}
\end{array}\right]\left[\begin{array}{l}
C \\
D
\end{array}\right]=C^{2}-D^{2}=I_{n}
$$

we obtain

$$
\left[\begin{array}{l}
C \\
D
\end{array}\right]^{\#}=I_{n}\left[\begin{array}{ll}
C & D
\end{array}\right]\left(J_{2} \otimes I_{n}\right)
$$

Thus,

$$
\left[\begin{array}{l}
C \\
D
\end{array}\right]^{\#}\left(B \otimes I_{n}\right)\left[\begin{array}{l}
C \\
D
\end{array}\right]=\left[\begin{array}{ll}
C & D
\end{array}\right]\left[\begin{array}{cc}
\lambda_{1} I_{n} & 0 \\
0 & -\lambda_{2} I_{n}
\end{array}\right]\left[\begin{array}{l}
C \\
D
\end{array}\right]=\lambda_{1} C^{2}-\lambda_{2} D^{2}=A .
$$

Let us assume now that $W(A) \subseteq W_{J_{2}}^{-}(B)$. Since $\left.\left.W_{J_{2}}^{-}(B)=\right]-\infty, \lambda_{2}\right]$, we easily conclude that $A$ is Hermitian and the matrices $\left(A-\lambda_{1} I_{n}\right),\left(A-\lambda_{2} I_{n}\right)$ are negative semidefinite.

Let define $C$ and $D$ as follows:

$$
C=\sqrt{\frac{\lambda_{2} I_{n}-A}{\lambda_{1}-\lambda_{2}}}, \quad D=\sqrt{\frac{\lambda_{1} I_{n}-A}{\lambda_{1}-\lambda_{2}}} .
$$

Having in mind that

$$
\left[\begin{array}{ll}
C & D
\end{array}\right]\left(J_{2} \otimes I_{n}\right)\left[\begin{array}{l}
C \\
D
\end{array}\right]=\left[\begin{array}{ll}
C & D
\end{array}\right]\left[\begin{array}{cc}
I_{n} & 0 \\
0 & -I_{n}
\end{array}\right]\left[\begin{array}{l}
C \\
D
\end{array}\right]=C^{2}-D^{2}=-I_{n},
$$

we get

Henceforth,

$$
\left[\begin{array}{l}
C \\
D
\end{array}\right]^{\#}=-I_{n}\left[\begin{array}{ll}
C & D
\end{array}\right]\left(J_{2} \otimes I_{n}\right)
$$

$$
\left[\begin{array}{l}
C \\
D
\end{array}\right]^{\#}\left(B \otimes I_{n}\right)\left[\begin{array}{l}
C \\
D
\end{array}\right]=-\left[\begin{array}{ll}
C & D
\end{array}\right]\left[\begin{array}{cc}
\lambda_{1} I_{n} & 0 \\
0 & -\lambda_{2} I_{n}
\end{array}\right]\left[\begin{array}{l}
C \\
D
\end{array}\right]=-\lambda_{1} C^{2}+\lambda_{2} D^{2}=A
$$


Theorem 6.2. Let

$$
B=\operatorname{diag}\left(\lambda_{1}, \lambda_{2}\right), \lambda_{1}>\lambda_{2}
$$

If $A$ satisfies $W_{J}(A) \subseteq W_{J_{2}}(B)$ for $J=I_{r} \oplus-I_{n-r}=\operatorname{diag}\left(\epsilon_{1}, \ldots, \epsilon_{n}\right)$, then $A$ can be dilated to an operator of the form $B \otimes I_{n}$.

Proof. According to the hypothesis, $\left.\left.W_{J_{2}}(B)=\right]-\infty, \lambda_{2}\right] \cup\left[\lambda_{1},+\infty\left[\right.\right.$. Hence $W_{J}(A)$ is a subset of the real line, and so $A$ is $J$-Hermitian. Further, $A$ is $J$-unitarily diagonalizable, otherwise $W_{J}(A)$ would be the whole real line. Assume firstly that $A=\operatorname{diag}\left(\mu_{1}, \ldots, \mu_{n}\right)$, with $\mu_{1} \geq \mu_{2} \geq \ldots \geq \mu_{n}$. Since $W_{J}(A) \subseteq W_{J_{2}}(B)$, we infer that $\sigma_{J}^{+}(A)=\left\{\mu_{1}, \ldots, \mu_{r}\right\}$ and $\sigma_{J}^{-}(A)=\left\{\mu_{r+1}, \ldots, \mu_{n}\right\}$ and so we have $\mu_{1} \geq \ldots \geq \mu_{r} \geq \lambda_{1}>\lambda_{2} \geq \mu_{r+1} \geq \ldots \geq \mu_{n}$. Given

$$
B \otimes I_{n}=\left[\begin{array}{cc}
\lambda_{1} I_{n} & 0 \\
0 & \lambda_{2} I_{n}
\end{array}\right], \quad J_{2} \otimes I_{n}=\left[\begin{array}{cc}
I_{n} & 0 \\
0 & -I_{n}
\end{array}\right],
$$

we search an isometric matrix $V=\left[P^{T} Q^{T}\right]^{T}$ such that

$$
\left[\begin{array}{ll}
P^{*} & Q^{*}
\end{array}\right] J_{2} \otimes I_{n}\left[\begin{array}{l}
P \\
Q
\end{array}\right]=P^{*} P-Q^{*} Q=J,
$$

and

$$
\left[\begin{array}{ll}
P^{*} & Q^{*}
\end{array}\right]\left(J_{2} \otimes I_{n}\right)\left(B \otimes I_{n}\right)\left[\begin{array}{l}
P \\
Q
\end{array}\right]=\lambda_{1} P^{*} P-\lambda_{2} Q^{*} Q=J A .
$$

Thus,

$$
\begin{aligned}
& P^{*} P=J+Q^{*} Q, \\
& Q^{*} Q=\frac{J\left(A-\lambda_{1} I_{n}\right)}{\lambda_{1}-\lambda_{2}}=\frac{\operatorname{diag}\left(\epsilon_{1}\left(\mu_{1}-\lambda_{1}\right), \ldots, \epsilon_{n}\left(\mu_{n}-\lambda_{1}\right)\right)}{\lambda_{1}-\lambda_{2}}, \\
& P^{*} P=\frac{J\left(A-\lambda_{2} I_{n}\right)}{\lambda_{1}-\lambda_{2}}=\frac{\operatorname{diag}\left(\epsilon_{1}\left(\mu_{1}-\lambda_{2}\right), \ldots, \epsilon_{n}\left(\mu_{n}-\lambda_{2}\right)\right)}{\lambda_{1}-\lambda_{2}} .
\end{aligned}
$$

Noticing that $\epsilon_{j}\left(\mu_{j}-\lambda_{k}\right) \geq 0, j=1, \ldots, n, k=1,2$, let us define

$$
\begin{aligned}
& Q=\sqrt{\frac{\operatorname{diag}\left(\epsilon_{1}\left(\mu_{1}-\lambda_{1}\right), \ldots, \epsilon_{n}\left(\mu_{n}-\lambda_{1}\right)\right)}{\lambda_{1}-\lambda_{2}},} \\
& P=\sqrt{\frac{\operatorname{diag}\left(\epsilon_{1}\left(\mu_{1}-\lambda_{2}\right), \ldots, \epsilon_{n}\left(\mu_{n}-\lambda_{2}\right)\right)}{\lambda_{1}-\lambda_{2}} .}
\end{aligned}
$$

If $A$ is not in diagonal form, a $J$-unitary matrix can be found such that $A=$ $U^{*} \operatorname{diag}\left(\mu_{1}, \ldots, \mu_{n}\right) U$. Then, replace $V$ by $W=V U$ and the theorem follows by the first part of the proof.

Theorem 6.3. Let

$$
\begin{aligned}
& B=\operatorname{diag}(1, i, 0), J_{3}=\operatorname{diag}(1,1,-1), \\
& A=\operatorname{diag}\left(\alpha_{1}+i \beta_{1}, \ldots, \alpha_{n}+i \beta_{n}\right)=H+i K, \alpha_{j}, \beta_{j} \in \mathbb{R}, j=1, \ldots, n .
\end{aligned}
$$

If $W_{J}(A) \subseteq W_{J_{3}}(B)$ for $J=I_{r} \oplus-I_{n-r}=\operatorname{diag}\left(\epsilon_{1}, \ldots, \epsilon_{n}\right)$, then $A$ can be dilated to an operator of the form $B \otimes I_{n}$. 
Proof. Under the conditions of the theorem, $W_{J_{3}}(B)$ is the pseudo-convex hull of the points $1, i, 0$, that is, the least (in the sense of set containment) pseudoconvex set containing these points [7, Proposition 2.3]. Since according to the hypothesis $W_{J}(A) \subseteq W_{J_{3}}(B)$, and the projection of $W_{J}^{ \pm}(A)$ on the real axis is given by $W_{J}^{ \pm}(H)$, we may conclude that

$$
\left.\left.W_{J}^{-}(H) \subseteq\right]-\infty, 0\right]
$$

and

$$
W_{J}^{+}(H) \subseteq[1,+\infty[\text {. }
$$

Henceforth, we have $\alpha_{1} \geq \ldots \geq \alpha_{r} \geq 1>0 \geq \alpha_{r+1} \geq \ldots \geq \alpha_{n}$. By a similar reasoning, but considering the projection of $W_{J}^{ \pm}(A)$ on the imaginary axis, we conclude that

$$
W_{J}^{+}(K) \subseteq[0,+\infty[
$$

and

$$
\left.\left.W_{J}^{-}(H) \subseteq\right]-\infty, 0\right]
$$

Hence $\beta_{1} \geq \ldots \geq \beta_{r} \geq 0 \geq \beta_{r+1} \geq \ldots \geq \beta_{n}$. Considering

$$
B \otimes I_{n}=\left[\begin{array}{ccc}
I_{n} & 0 & 0 \\
0 & i I_{n} & 0 \\
0 & 0 & 0
\end{array}\right], \quad J_{3} \otimes I_{n}=\left[\begin{array}{ccc}
I_{n} & 0 & 0 \\
0 & I_{n} & 0 \\
0 & 0 & -I_{n}
\end{array}\right]
$$

we search an isometric matrix $V=\left[P^{T} Q^{T} R^{T}\right]^{T}$ such that

$$
\begin{aligned}
& {\left[\begin{array}{lll}
P^{*} & Q^{*} & R^{*}
\end{array}\right] J_{3} \otimes I_{n}\left[\begin{array}{l}
P \\
Q \\
R
\end{array}\right]=P^{*} P+Q^{*} Q-R^{*} R=J} \\
& {\left[\begin{array}{lll}
P^{*} & Q^{*} & R^{*}
\end{array}\right]\left(J_{3} \otimes I_{n}\right)\left(B \otimes I_{n}\right)\left[\begin{array}{l}
P \\
Q \\
R
\end{array}\right]=P^{*} P+i Q^{*} Q=J A .}
\end{aligned}
$$

Thus,

$$
\begin{aligned}
& P^{*} P+Q^{*} Q=J+R^{*} R \\
& P^{*} P=J H=\operatorname{diag}\left(\epsilon_{1} \alpha_{1}, \ldots, \epsilon_{n} \alpha_{n}\right), \\
& Q^{*} Q=J K=\operatorname{diag}\left(\epsilon_{1} \beta_{1}, \ldots, \epsilon_{n} \beta_{n}\right), \\
& R^{*} R=\operatorname{diag}\left(\epsilon_{1}\left(\alpha_{1}+\beta_{1}-1\right), \ldots, \epsilon_{n}\left(\alpha_{n}+\beta_{n}-1\right)\right) .
\end{aligned}
$$

Observing that, under the hypothesis $\epsilon_{j} \alpha_{j} \geq 0, \epsilon_{j} \beta_{j} \geq 0$ and $\epsilon_{j}\left(\alpha_{j}+\beta_{j}-1\right) \geq 0$ for $j=1, \ldots, n$, we can define the positive semi-definite matrices $P, Q, R$ as follows:

$$
\begin{aligned}
P & =\sqrt{\operatorname{diag}\left(\epsilon_{1} \alpha_{1}, \ldots, \epsilon_{n} \alpha_{n}\right)}, \\
Q & =\sqrt{\operatorname{diag}\left(\epsilon_{1} \beta_{1}, \ldots, \epsilon_{n} \beta_{n}\right)}, \\
R & =\sqrt{\operatorname{diag}\left(\epsilon_{1}\left(\alpha_{1}+\beta_{1}-1\right), \ldots, \epsilon_{n}\left(\alpha_{n}+\beta_{n}-1\right)\right)} .
\end{aligned}
$$




\section{REFERENCES}

1. T. Ando, Structure of operators with numerical range one, Acta Sci. Math. (Szeged) 34 (1973), 11-15.

2. T. Ando and C.-K. Li (ed.), The Numerical Range and Numerical Radii, Linear Multilinear Algebra, 37 (1994), no. 1-3, Gordon and Breach Science Publishers, Yverdon, 1994. pp. $1-238$.

3. T. Bayasgalan, The numerical ranges of linear operators in spaces with an indefinite metric (Russian), Acta Math. Hungar. 57 (1991), 7-9.

4. N. Bebiano, J. da Providência and A. Nata, The numerical range of banded 2-Toeplitz operators in spaces with an indefinite metric, Linear Multilinear Algebra, in press, DOI:10.1080/03081087.2013.769100.

5. N. Bebiano and J. da Providência, Numerical ranges of quadratic operators in spaces with an indefinite metric, Electron. J. Linear Algebra (to appear).

6. N. Bebiano, R. Lemos, J. da Providência and G. Soares, On generalized numerical ranges of operators on an indefinite inner product space, Linear Multilinear Algebra 52 (2004), 203-233.

7. N. Bebiano, R. Lemos, J. da Providência and G. Soares, On the geometry of numerical ranges in spaces with an indefinite inner product, Linear Algebra Appl. 399 (2005), 17-34.

8. N. Bebiano, J. da Providência, A. Nata and G. Soares, Krein spaces numerical ranges and their computer generation, Electron. J. Linear Algebra 17 (2008), 192-208.

9. M.-T. Chien, Lina Yeh, Y.-T. Yeh and F.-Z. Lin, On geometric properties of the numerical range, Linear Algebra Appl. 274 (1998), 389-410.

10. I. Gohberg, P. Lancaster and L. Rodman, Matrices and indefinite scalar product, Birkhäuser, Basel-Boston, 1983.

11. R.A. Horn and C.R. Johnson, Topics in Matrix Analysis, Cambridge University Press, New York, 1991.

12. C.-K. Li, N.K. Tsing and F. Uhlig, Numerical ranges of an operator on an indefinite inner product space, Electron. J. Linear Algebra 1 (1996), 1-17.

13. C.-K. Li and L. Rodman, Shapes and computer generation of numerical ranges of Krein space operators, Electron. J. Linear Algebra 3 (1998), 31-47.

14. C.-K. Li and L. Rodman, Remarks on numerical ranges of operators in spaces with an indefinite inner metric, Proc. Amer. Math. Soc. 126 (1998), 973-982.

15. Man-Duen Choi and C.-K.Li, Numerical Ranges and Dilations, Linear Multilinear Algebra 47 (2000), 35-48.

16. M. Marcus and C. Pesce, Computer generated numerical ranges and some resulting theorems, Linear Multilinear Algebra 20 (1987), 121-157.

17. B.A. Mirman, Numerical range and norm of a linear operator, Vorone z. Gos. Uni. Trudy Sem. Funckional Anal. 10 (1968), 51-55.

${ }^{1}$ CMUC, University of Coimbra, Department of Mathematics, P 3001-454 CoimBra, Portugal.

E-mail address: bebiano@mat.uc.pt

2 CFC, University of Coimbra, Department of Physics, P 3004-516 Coimbra, PORTUGAL.

E-mail address: providencia@teor.fis.uc.pt 